\title{
Immunomodulatory Properties of Composite Materials Based on Polylactide and Hydroxyapatite
}

\author{
Kurzina $\mathbf{I}^{1}$, Churina $\mathrm{Y}^{1,2 *}$, Shapovalova $\mathbf{Y}^{1}$, Syusyukina $\mathbf{V}^{1}$ and Kzhyshkowska $\mathrm{J}^{1,3}$ \\ ${ }^{1}$ National Research Tomsk State University, Tomsk, Russia \\ ${ }^{2}$ Siberian State Medical University, Tomsk, Russia \\ ${ }^{3}$ Institute of Transfusion Medicine and Immunology, University of Heidelberg, Heidelberg, Germany
}

\begin{abstract}
In the present study composites based on polylactide and hydroxyapatite with the content of components 50:50 and $75: 25$ were investigated. Components were mixed at $40^{\circ} \mathrm{C}$, which was followed by the sonication procedures and its precipitation in ethanol. The analysis of the following composite materials revealed that their chemicalcrystallographic characteristics of individual components remained intact after varying its dispersion and material crystallinity degree. Composite material of the ratio 75:25 were characterized by the lowest degree of crystallinity - $20.5 \%$ and the average crystallite size up to $28.8 \mathrm{~nm}$ showed an increased roughness and dispersive component of surface energy. In comparison to polylactide, the composite has a high capacity for osseointegration. In the paper, special attention is given to the immunomodulatory properties of composite materials. Assessment of the immune system cells showed that the macrophages are most viable in the presence of pure polylactide and composite $75 / 25$. Intensive secretion of proinflammatory cytokines in macrophage cultures in vitro was not found at that.
\end{abstract}

\section{Highlights}

- An express method of producing polymeric composites based on hydroxyapatite and polylactide was proposed

- The mass ratio of the components affects the formation of the calcium phosphate layer in the model SBF solution

- Reduction of the content of hydroxyapatite in the polymer matrix helps to increase the viability of macrophages in vitro

- The resulting composite materials do not cause the expression of pro-inflammatory cytokines

Keywords: Polylactide; Hydroxyapatite; Composite material; Immune response; Bioactive composites

\section{Introduction}

Many medical challenges that involve treatment of damaged tissues can be solved by a regenerative approach. Such approach is mastered by placing the implant materials in a damaged area/injury site, which would promote natural excretion followed by the new healthy tissue formation in its place. Foreign body response to the implant material is greatly influenced by the immune status of a patient; this is in addition to physical and chemical characteristics of the implant materials itself $[1,2]$. It is very critical that materials, which are used in the regenerative approach, have high biocompatibility, controlled rate of bioresorption, porosity, sufficient for the flow of body fluids and for cell penetration and proper vascularization of the ingrown tissue $[3,4]$. There are various materials, which are used in implantation procedures; the three major types are metals, polymers and ceramics. Almost all of their subtypes initiate some level of an inflammatory response becoming an ultimate cause for rejection of the implants. Currently in medicine metals [5-7], ceramics, bioactive glass [8-11], polymer materials [12-14] are used as bone tissue substitutes. Use of polymer composite materials along with the combination characteristics of all initial components is the most promising approach [15-17]. Among the synthetic biodegradable polymer materials, polylactide (PL) is believed to be the most famous one. This is due to the ability of PL to possess excellent biodegradable and biocompatible characteristics [1821]. A combination of hydroxyapatite (HA) [22-25] and PL provides implant materials with greater natural tissue characteristics. It was shown by different science groups that HA and PL based composites combine most necessary properties of the source materials and reveal new biophysical and chemical characteristics that have a positive effect on the functional/regenerative properties of the implants [26]. Nowadays there are multiple approaches available in the methods material obtainment $[27,28]$. The functional properties of the material products are determined by its initial composition and their method of production.

Using the above-mentioned tactics, polylactic acid composites containing hydroxyapatite fibers were obtained by the team of scientists [29]. Ceramic-polymer composites were prepared by hotpressing a mixture that composed of PL and HA fibers. The modulus of elasticity was improved effectively, even by introducing small amounts of HA. In this study Diao et al. [30], melt blending was used to fabricate poly (lactic acid)/hydroxyapatite nanocomposites. Also they used nanocomposite film which was based on surface modifying HA nanoparticles with dodecyl alcohol. This nanocomposite demonstrated good cartilage cell attachment, spreading and proliferation.

The method of grafting ring-opening polymerization of L-lactide on the surface of hydroxyapatite nanoparticles was described by the researchers [31,32]. PL was directly connected to the HA surface via the chemical linkage. The mechanical properties of the obtained materials from the study are close to the properties of natural bone, but, nevertheless, are not ideal for implantation, because the composition and biocompatibility have not been studied well. However, the described characteristics are key ones for the bone substitutes. The mode

*Corresponding author: Churina Y, MD, Doctor-Immunologist, Professor of the National Research Tomsk State University, 36, Lenin ave., Tomsk, 634050, Russia, Tel: +7-913-806-0700; E-mail: lena1236@yandex.ru

Received February 13, 2018; Accepted February 20, 2018; Published February 26, 2018

Citation: Kurzina I, Churina Y, Shapovalova Y, Syusyukina V, Kzhyshkowska J (2018) Immunomodulatory Properties of Composite Materials Based on Polylactide and Hydroxyapatite. Bioceram Dev Appl 8: 109. doi: 10.4172/2090-5025.1000109

Copyright: $\odot 2018$ Kurzina I, et al. This is an open-access article distributed under the terms of the Creative Commons Attribution License, which permits unrestricted use, distribution, and reproduction in any medium, provided the original author and source are credited. 
of production, composition, molding and sterilization of composite materials has great influence on physico-chemical properties as they also affect a large range of biological factors. Despite already existing PL and HA based methods of composites production, the technique of obtainment and an appropriate combination of components, which allows creating materials identical to the bone tissue, still has not been developed. Consequently, it is believed that finding optimal obtainment methods, studying the structure of the materials itself and investigating biophysical characteristics of the composites, which depend on conditions of production, are a top priority for researchers.

In this work, the authors employ the synthetic hydroxyapatite materials, which have been obtained by the method of microwave synthesis. The studied materials were characterized by all the necessary morphological and structural-phase properties. The authors' approach in the methods of material obtainment allows retaining the individual properties of the composite's initial components. The aim of this investigation is obtaining influence of the preparation's methods and elemental-phase composition on the material formation with a necessary complex of biochemical and structural-chemical characteristics for bone tissue regeneration.

\section{Materials}

Polylactide was synthesized from L-lactide [33] by open-ring polymerization in the presence of tin octanoate as catalyst. PL was dissolved in chloroform at $40^{\circ} \mathrm{C}$; the resulting solution was dropwise added to a fivefold excess of ethyl alcohol. Then PL was precipitated in ethanol in a form of white opaque fibers. The purified PL mixture was dried in a drying box at $40^{\circ} \mathrm{C}$. Hydroxyapatite was obtained by the method of liquid-phase synthesis at $\mathrm{pH} \sim 11$ using microwave radiation [34]. Composites 1, 2 were obtained by mixing the solution with polylactide in chloroform $(c=0.050 \pm 0.01 \mathrm{~g} / \mathrm{ml})$ and hydroxyapatite powder in mass ratios of 50:50 and 75:25, respectively. The suspension was exposed to ultrasound with a frequency of $40 \mathrm{kHz}$ for 20 minutes and then with constant stirring precipitated in five-fold excess of cold ethyl alcohol. The obtained opaque fibers of white color were dried in a drying box at the temperature of $40^{\circ} \mathrm{C}$ to the constant weight. Then the fibers were crushed and molded into the form of tablets $(\mathrm{m}=0.20 \pm 0.01$ g) on the laboratory hydraulic press PGR-10 under the pressure of 200 bar; the tablet diameter is $19 \mathrm{~mm}$, thickness- $1 \mathrm{~mm}$.

\section{Methods}

\section{Definition of the elemental and phase composition}

The molecular weight of polylactic acid was determined by gel permeation chromatography using HPLC chromatograph UltiMate 3000 (Dionex, USA). Chloroform was used as an eluent. FTIR Nicolet 6700 infrared spectrometer was used to characterize PL, HA, composites 1,2 in the range of $4000-500 \mathrm{~cm}^{-1}$. Identification of the absorption bands was carried out by the characteristic frequencies. To verify HA phase in composite materials, the phase analysis was performed using X-ray diffraction (XRD). The Shimadzu XRD 6000 system was operated at $40 \mathrm{kV}$ and $30 \mathrm{~mA}$ at a $2 \theta$ range of $10^{\circ}-80^{\circ}$, using a step size of 0.02 with a $0.60 \mathrm{sec}$ exposure time.

\section{Morphological and structural characteristics}

Morphology of the samples' surface was determined by scanning electron microscope Hitachi TM-3000 operating at an acceleration voltage of $15 \mathrm{kV}$. Estimation of the parameters of the porous structure and the specific surface of the materials was performed using an automatic gas adsorption analyzer 3Flex produced by Micromeritics
(USA). Surface characteristics were defined by the BET methods. The roughness was measured on the Profilometer-296 on parameter Ra. For evaluation of the wettability, the research was carried out using DSA1 software on the installation Easy Drop (KRUSS).

\section{Evaluation of biocompatibility in vitro}

For evaluation of the composite capacity to form the new calcium phosphate layer (CPL) on its surface, the SBF-research was carried out [35]. For this study, the substrates formed from PL, composites 1, 2 were placed in the SBF solution, which by its mineral composition and ion concentration is identical to human blood plasma. The scaffolds were kept in the SBF solution at $37^{\circ} \mathrm{C}$ during 22 days with daily update of the solution; after holding in the solution, the surface of the substrates were studied by the SEM method.

The human immune system cells' reaction was investigated using the cell-mediated immune response in monocytes CD14+. Before the investigation, the samples were sterilized by soaking in ethanol (70\%) for 30 minutes and UV irradiation. Isolated cells were resuspended in ex-vivo cell medium (with Dexamethasone at $1 \times 10^{-8} \mathrm{M}$, M-CSF $5 \mathrm{ng}$ / $\mathrm{ml}$ ) at a concentration of $1 \times 10^{6}$ cells per ml. The cells were seeded in 12 well plates and stimulated with desired cytokines: INF- $\gamma$ at $100 \mathrm{ng} / \mathrm{ml}$, IL- 4 at $10 \mathrm{ng} / \mathrm{ml}$. Cells were incubated in the Macrophage serum-free medium or ex-vivo at $37^{\circ} \mathrm{C}$ with $7.5 \% \mathrm{CO}_{2}$ for 6 days.

Concentration of human IL-6, CCL18, TNF- $\alpha$ (R\&D Systems, Germany) levels in cell culture supernatants was measured according to the manufacturer's instructions. All samples were analyzed in duplicates. Assessment of cells viability after 6 days of culturing was carried out using optical microscopy and the intensity of the fluorescence signal of living cells after the interaction with AlamarBlue.

\section{Conformity with ethical principles}

The clinical study was carried out in accordance with the World Medical Association Declaration of Helsinki "Ethical Principles for Medical Research Involving Human Subjects" amended in 2000 and "Clinical practice rules in the Russian Federation" ratified by the Decree of the Ministry of Health of the Russian Federation of 19.06.2003, No. 266. All patients signed the informed consent to the participation in the research in accordance with the record, approved by the local ethic committee affiliated to the Science and Engineering Board of Tomsk State University.

\section{Results}

\section{Physical and chemical characteristics of materials}

Figure 1 represents XRD patterns of samples. Strong peaks were observed at $16.8^{\circ}$ and $19^{\circ}$, which corresponds to PL. Peaks at $26^{\circ}$, $31.9^{\circ}, 32.9^{\circ}$ indicate the presence of the HA phase. The obtained $\mathrm{HA}$ is characterized by $100 \%$ crystallinity and crystallographically corresponds to $\mathrm{HA}\left(\mathrm{Ca}_{5}\left(\mathrm{PO}_{4}\right)_{3} \mathrm{OH}\right)$. Lattice parameters correspond to the table values; the coherent scattering region is $36.3 \mathrm{~nm}$.

The obtained polylactide with MM 100000 was characterized by a degree of crystallinity of $15 \%$ (Table 1 ). There were diffraction lines typical of the PL and HA in the XRD spectrum of the formed composites. The appearance of new reflexes and the shift of XRD lines turned out to be typical of the PL, and HA were not detected, which indicates the preservation of the chemical composition and crystallographic identity of the source components. However, mixing of the components during formation of composites led to a change in the material's crystallinity degree. At the mass ratio of components equal to 50:50, the intensity of 
Citation: Kurzina I, Churina Y, Shapovalova Y, Syusyukina V, Kzhyshkowska J (2018) Immunomodulatory Properties of Composite Materials Based on Polylactide and Hydroxyapatite. Bioceram Dev Appl 8: 109. doi: 10.4172/2090-5025.1000109

Page 3 of 7

the PL and HA bands was similar, which explains the homogeneity of mixing and stability of the composite structure. However, the material was characterized by a decrease in the coherent scattering region (CSR) of hydroxyapatite to $20 \mathrm{~nm}$, saving areas of crystallinity for PL. CSR of hydroxyapatite (at the ratio of PL and HA equal to 75:25) relatively decreased to its initial size of $28 \mathrm{~nm}$. The sample was $79.5 \%$ amorphous due to the PL crystallinity degree reduction. Therefore, introduction of the HA into the polymer matrix leads to its polydispersity increase as well as to a uniform distribution in the polymer. The HA particles in an amount up to $25 \mathrm{wt}$ \% could be an inhibiting factor in the formation of polymer microcrystals in the composite.

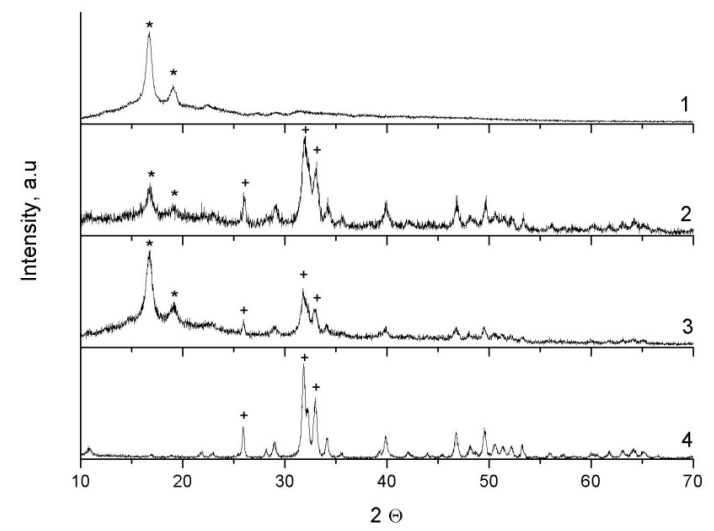

Figure 1: XRD patterns: 1- PL; 2- composites 2 (PL/HA=75/25); 3- composite 1 $(\mathrm{PL} / \mathrm{HA}=50 / 50)$; 4- HA; reflexes:- $\mathrm{PL},+\mathrm{+}-\mathrm{HA}$.

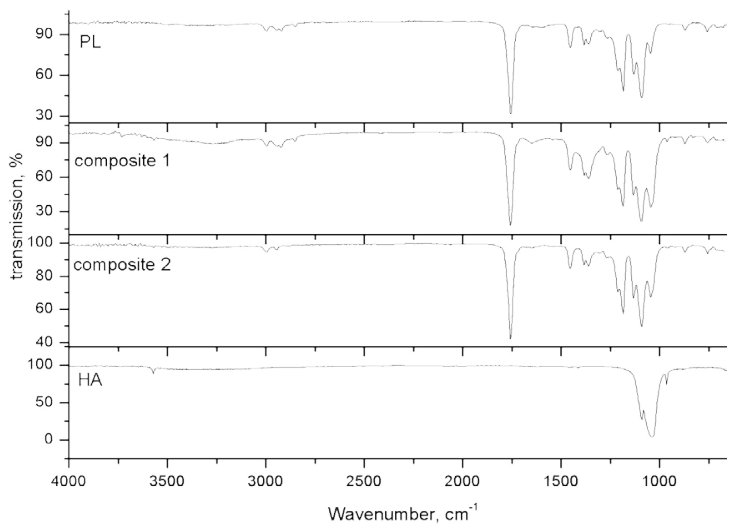

Figure 2: FT-IR spectra of PL, HA, composites 1-2.
The infrared spectra of PL, HA, composites 1, 2 are shown in Figure 2. IR spectroscopy characterizes chemical bonds in the material. The absorption bands of stretching (950-1200 $\left.\mathrm{cm}^{-1}\right)$, deformation (560$\left.610 \mathrm{~cm}^{-1}\right)$ vibrations of phosphate groups and stretching $\left(3570 \mathrm{~cm}^{-1}\right)$ vibrations of hydroxyl-groups in the IR-spectrum are characteristic for HA. The stretching vibrations of carbonyl groups in PL spectra are registered as $1730-1750 \mathrm{~cm}^{-1}$ bands. In the spectra of the composites, the absorption bands characteristic for HA and for PL are detected. It has been found that the composites do not form new chemical bonds between hydroxyapatite and polylactide.

Formation of the internal structure goes at the expense of hydrogen and donor-acceptor bonding between the particles of HA and PL. Redistribution of chemical bonds in the material leads to the change in surface energy.

The method of obtainment and the ratio of components in composite materials influence the surface characteristics of the samples. Increasing the proportion of polymer in the sample decreases the specific surface. The polylactide is formed from irregularly shaped particles with an average size of $2-4 \mathrm{~mm}$, which plays a role in the physics-chemistry of surface samples. HA is a powder with an average particle size of 50-70 $\mathrm{nm}$, which are easily compactable, roughness is $1.32 \mathrm{~nm}$. In the process of obtainment and formation of the composite materials the HA particles are located between the globules of the $\mathrm{PL}$, resulting in an increase in roughness relatively to the initial materials due to the protruding shards of PL crystals. The roughness of composites 1,2 is 7.24 and $7.61 \mu \mathrm{m}$ (Table 1). It should be noted that the composite with the high content of PL was characterized by the highest roughness.

The chemical composition and surface roughness determined the degree of hydrophilicity of the material and the level of surface energy (Table 1). All materials are hydrophilic (contact angle is less than 90 degrees). The study of the hydrophilicity degree showed that polylactide was well moistened both by water and by glycerol, has a high surface energy in comparison with composites and hydroxyapatite. The introduction of HA to polylactide leads to the sharp increase of the wetting angle (composite 2), which by increasing the proportion of filler in the polymer matrix decreases again. With the increase of polymer concentration, the increase in the dispersive component of total surface energy was observed, which indicated the predominance of low energy interaction in the material.

\section{Assessment of biodegradation and immunomodulating ac- tivity in vitro}

Physical and chemical properties of the material surface determine the behavior of composites in the biological environment. The chemical

\begin{tabular}{|c|c|c|c|c|c|c|c|c|c|}
\hline Sample & Phases & $\begin{array}{c}\text { Phase.mass, } \\
\%\end{array}$ & $\begin{array}{l}\text { Coherent } \\
\text { scattering } \\
\text { region, } \\
\text { nm }\end{array}$ & $\begin{array}{c}\text { Dispersion } \\
\text { surface } \\
\text { energy } \sigma_{\mathrm{d}} \\
\mathrm{mJ} / \mathrm{m}^{2}\end{array}$ & $\begin{array}{c}\text { Polar surface } \\
\text { energy } \sigma_{p} \\
m J / m^{2}\end{array}$ & $\begin{array}{c}\text { Surface } \\
\text { energy, } \\
\mathrm{mJ} / \mathrm{m}^{2}\end{array}$ & $\begin{array}{c}\text { Roughness } \\
\text { coefficient } R_{\mathrm{a}} \\
\mu \mathrm{m}\end{array}$ & $\begin{array}{c}\text { Surface area } \\
\mathbf{S}_{\mathrm{a}^{\prime}} \\
\mathbf{g} / \mathrm{m}^{2}\end{array}$ & $\begin{array}{c}\text { Porosity, } \\
\text { nm }\end{array}$ \\
\hline \multirow{2}{*}{ Polylactide } & $\left(\mathrm{C}_{9} \mathrm{H}_{14} \mathrm{O}_{7}\right) \mathrm{n}$ & 15 & 12,7 & $0.58 \pm 0.15$ & $72.15 \pm 1.56$ & $72.74 \pm 1.72$ & 6.26 & - & - \\
\hline & Amorphous phase & 85 & - & - & - & - & - & - & - \\
\hline \multirow{3}{*}{ Composite 1} & $\mathrm{Ca}_{5}\left(\mathrm{PO}_{4}\right)_{3}(\mathrm{OH})$ & \multirow{2}{*}{44} & 20 & \multirow{3}{*}{$14.26 \pm 0.58$} & \multirow{3}{*}{$42.67 \pm 0.97$} & \multirow{3}{*}{$56.93 \pm 1.56$} & \multirow{3}{*}{7.24} & \multirow{3}{*}{25.1} & \multirow{3}{*}{31.3} \\
\hline & $\left(\mathrm{C}_{9} \mathrm{H}_{14} \mathrm{O}_{7}\right) \mathrm{n}$ & & 8,1 & & & & & & \\
\hline & Amorphous phase & 56 & - & & & & & & \\
\hline \multirow{3}{*}{ Composite 2} & $\mathrm{Ca}_{5}\left(\mathrm{PO}_{4}\right)_{3}(\mathrm{OH})$ & \multirow{2}{*}{20.5} & 28.8 & \multirow{3}{*}{$26.46 \pm 1.47$} & \multirow{3}{*}{$11.43 \pm 1.05$} & \multirow{3}{*}{$37.90 \pm 2.51$} & \multirow{3}{*}{7.61} & \multirow{3}{*}{9.0} & \multirow{3}{*}{23.7} \\
\hline & $\left(\mathrm{C}_{9} \mathrm{H}_{14} \mathrm{O}_{7}\right) \mathrm{n}$ & & 4,0 & & & & & & \\
\hline & Amorphous phase & 79.5 & - & & & & & & \\
\hline Hydroxyapatite & $\mathrm{Ca}_{5}\left(\mathrm{PO}_{4}\right)_{3}(\mathrm{OH})$ & 100 & 36.3 & $9.44 \pm 0.80$ & $58.79 \pm 2.24$ & $68.23 \pm 3.04$ & 1.32 & 106.0 & 20 \\
\hline
\end{tabular}

Table 1: Physico-chemical properties of materials. 
Citation: Kurzina I, Churina Y, Shapovalova Y, Syusyukina V, Kzhyshkowska J (2018) Immunomodulatory Properties of Composite Materials Based on Polylactide and Hydroxyapatite. Bioceram Dev Appl 8: 109. doi: 10.4172/2090-5025.1000109

composition, structural characteristics of composite materials affect the process of PL dissolution in the composite and the rate of samples bioresorption.

It is possible to evaluate the ability of the material to form a new surface layer of calcium phosphate by holding the samples in the SBFsolution. In the electronic micro photos of $\mathrm{PL}$, composites 1,2 were obtained by the SEM method before (Figures 3a-3c) and after (Figures 3d-3f) 4 weeks of holding in SBF, which indicates formation of a new calcium phosphate layer on the samples surface.

An active, but inhomogeneous, growth of new CPL lies on the surface of the pure PL, which is explained by a lack of chemical affinity to calcium phosphates. Composite 1 compared to PL and composite 2 has the least ability for ingrowth and osseointegration. In comparison to the PL, the major difference is that composites 1,2 are more active formation of the new layer on the composite 2 surface. New formed calcium phosphate is uniformly distributed on the surface of composite 2, which was confirmed by the EDRS data (Figure 4); the size of grains of spherical shape reached 6-7 $\mu \mathrm{m}$.
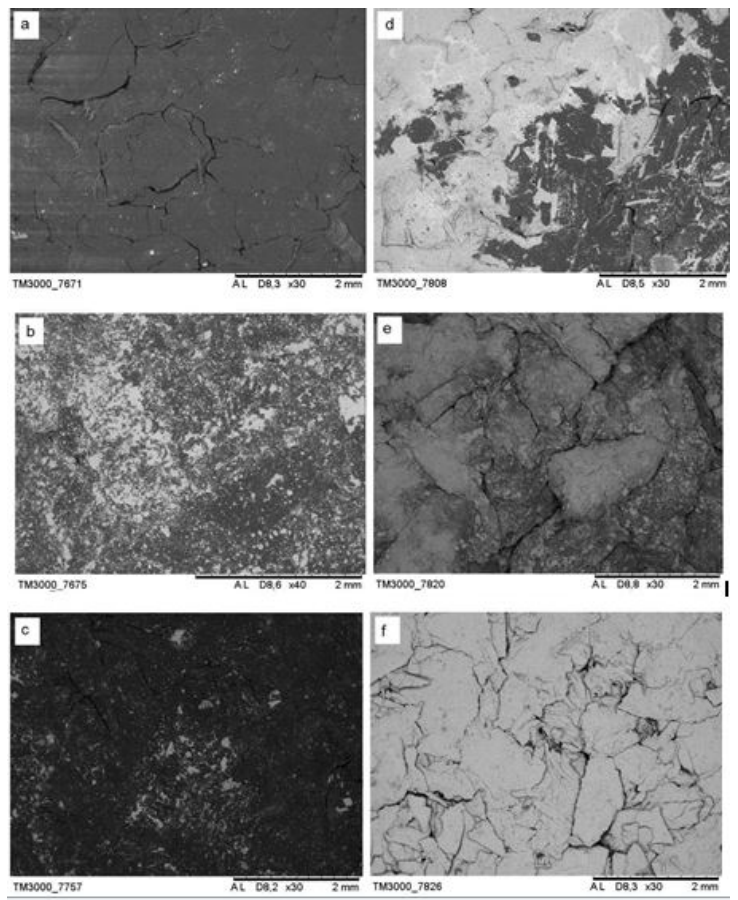

Figure 3: SEM images of PL (a, d), composite $1(b, e)$ and composite 2 (c, $f$ ) before (a, b, c) and after 4 weeks (d, e, f) in the SBF solution (30x).
The introduction of hydroxyapatite affected the rate of degradation of polymer matrix. The basis of the immune system reaction is a mechanism of the inflammatory cell-mediated immune response. Development of the immune inflammation in this case is associated with antigen of implant recognition by T-lymphocytes with subsequent activation of the innate immune factors, primarily humoral macrophages and their products. Evaluation of cells' cytotoxicity showed that the viability of macrophages in the presence of PL is retained at the control level. The interaction with hydroxyapatite caused death of the macrophages, which indicates that the HA has cytotoxic properties that cause cell damage and as a result suppression of the cellular immune response. In the presence of composite 2 in the case of donor 1-3, there is an increase in the number of living cells compared to control, which may be associated with the presence of certain immunomodulatory properties of this sample. On the other hand, composite 1 has a negative impact on immune system cells (Figure 5).

The direction of differentiation of peripheral blood monocytes to tissue macrophages and immunological functions, carried out by them subsequently, are determined by the nature of the antigen and its immunogenicity, local cytokine status of cells microenvironment. It occurs in three main ways: the classical pathway activation (M1activation), the alternative pathway activation (M2-activation) or the path of deactivating macrophages is realized. In the latter case, there is no immune response. Accordingly, the immune system response to the introduction of the implant is implemented by one of the possible ways- an inflammatory immune response, an inflammatory immune response or energy (tolerance).

M1 activation causes a cascade of immunological reactions that lead to the development of inflammation and tissue damage, which is accompanied by increased secretion of proinflammatory cytokines. M2-activation, in contrast, is caused by macrophages of the second type, the biological role of which is to regulate the intensity of the immune response, including by secreting inflammatory cytokines. This leads to increased angiogenesis with the subsequent repair process of tissue. In the case of monocytes differentiation into tolerogenic macrophages, energy with the participation of cytokines having suppressive activity is formed. Thus, the authors chose cytokines with proinflammatory properties (IL-6, TNF $\alpha$ ) as markers of M1-macrophage activation, inflammatory protein with immunosuppressive activity CCL18 as a marker of M2 activation [36]. The concentration of cytokines was determined by ELISA after 6 days of cells culturing in the presence of composites.

IL-6 is an action wide pro-inflammatory mediator involved in the induction of all complex local inflammation symptoms. IL-6 secretion by monocytes and macrophages immediately begins
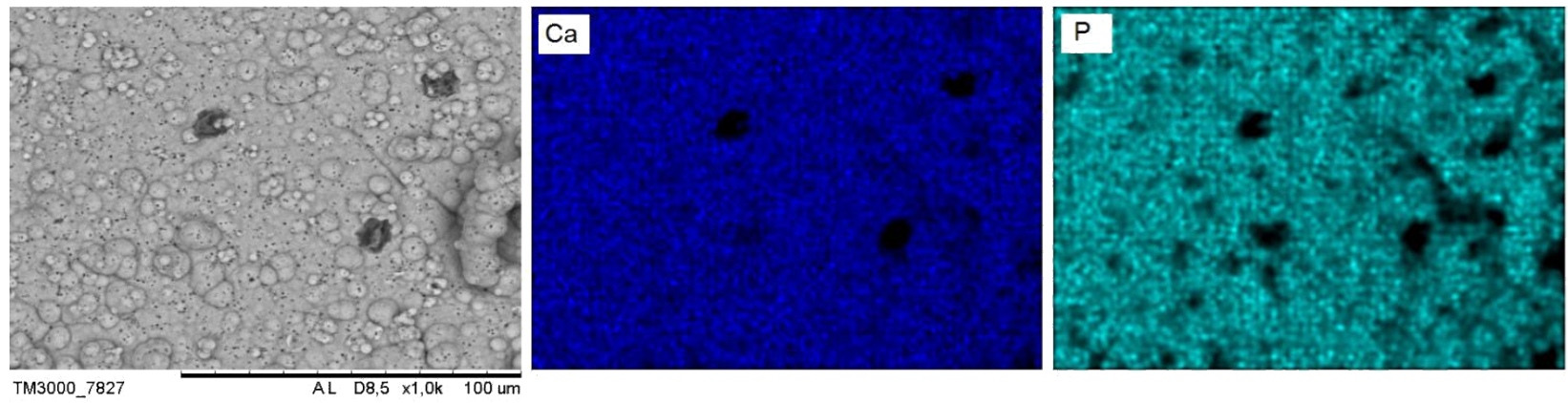

Figure 4: SEM image of composite 2 after 4 weeks in the SBF solution and distribution of $\mathrm{Ca}, \mathrm{P}$ on the surface (1000x). 
upon activation of cells by alien agents. IL- 6 affects the migration of phagocytes, increasing the production of CC-chemokines that attract monocytes and lymphocytes in the focus of inflammation. However, pro-inflammatory effects of IL-6 are less pronounced than that of IL-1 and TNF $\alpha$. In contrast, IL- 6 has the ability to inhibit the production of proinflammatory cytokines and chemokines by cells involved in the inflammatory process. Thus, IL- 6 can participate not only in the development, but also in limiting the inflammatory response.

The results of the ELISA showed that in the presence of polylactide without activation of macrophages (M0 macrophages), the expression of IL- 6 was observed in all donors. The highest level of a cytokine is from donor 3, which is associated with the presence of IL- 6 and in the control (Figure 6). During M2 activation of macrophages, the reduced levels of IL-6 are observed (Figure 7); during M1 activation there is a significant increase in the concentration of IL- 6 only from donor 4 (Figure 8). In the presence of composites 1,2, the high expression of

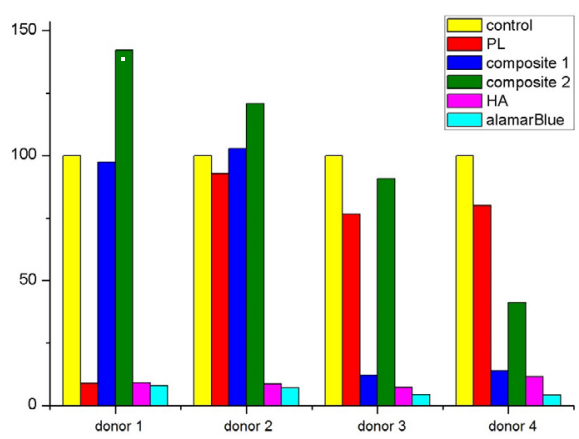

Figure 5: Cells viability after 6 days of cultivation in the presence of samples.

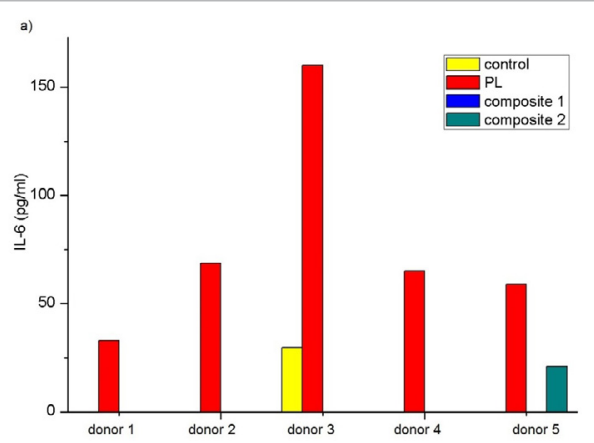

Figure 6: Effect of PL, composites 1, 2 on IL-6 production by primary human monocytes-derived macrophages: no stimulation.'

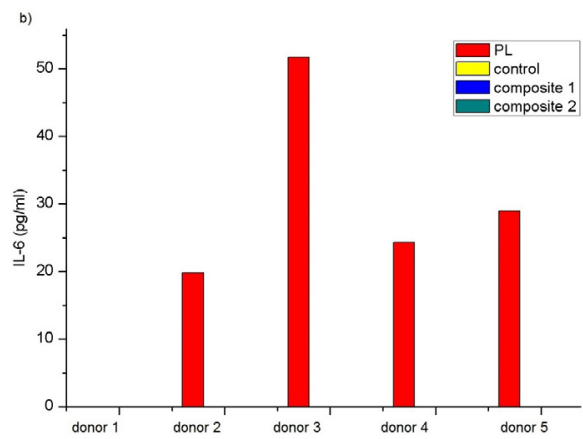

Figure 7: Effect of PL, composites 1, 2 on IL-6 production by primary human monocytes-derived macrophages: stimulation with IL-4.

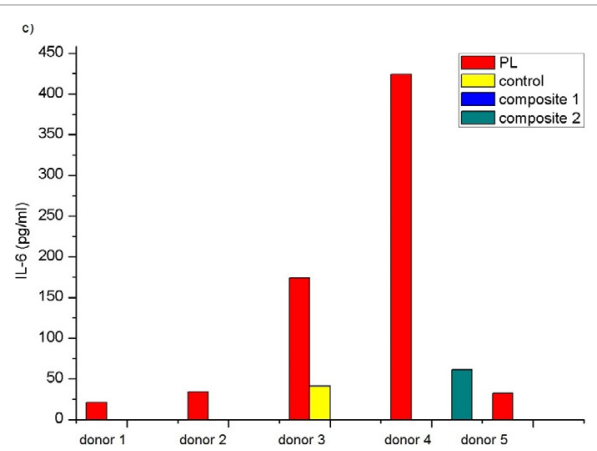

Figure 8: Effect of PL, composites 1, 2 on IL-6 production by primary human monocytes-derived macrophages: stimulation with IFNY.

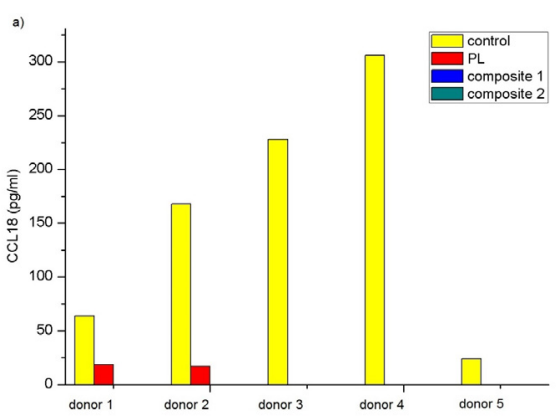

Figure 9: Effect of $\mathrm{PL}$, composites 1, 2 on CCL18 production by primary human monocytes-derived macrophages: no stimulation.

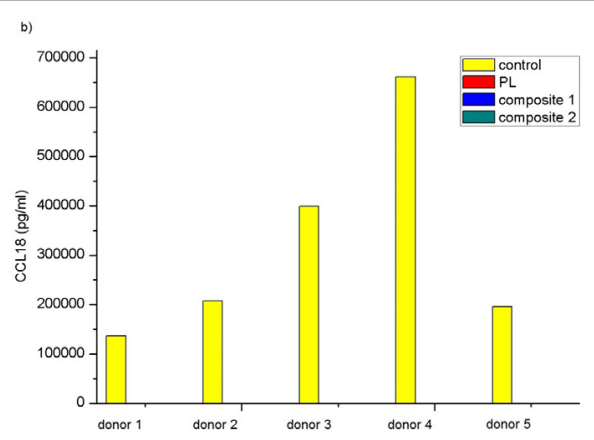

Figure 10: Effect of PL, composites 1, 2 on CCL18 production by primary human monocytes-derived macrophages: stimulation with IL-4.

IL-6 is not observed except donor 5 that indicates an absence of an acute inflammatory reaction of the immune system for these materials.

CCL18 is a protein belonging to the family of CC-chemokines. Initially, CCL18 was also known as AMAC-1 (alternative macrophage activation-associated CC chemokine-1), which is produced primarily by antigen-presenting cells of the innate immune system. CCL18 promotes the differentiation of monocytes into macrophages M2, which possess immunosuppressive and regulatory functions.

Immunoassay analysis showed that the CCL18 expression occurs in the control of all donors. Slight secretion of this cytokine in the presence of PL is observed in donors 1, 2 without macrophage activation (Figure 9 ) and in the case of activation of donor 2 by M1 (Figures 10 and 11). In the presence of composites 1, 2 and stimulation with IL-4, the expression of CCL18 is not observed (Figure 11), which indicates the absence of alternative activation of macrophages. 


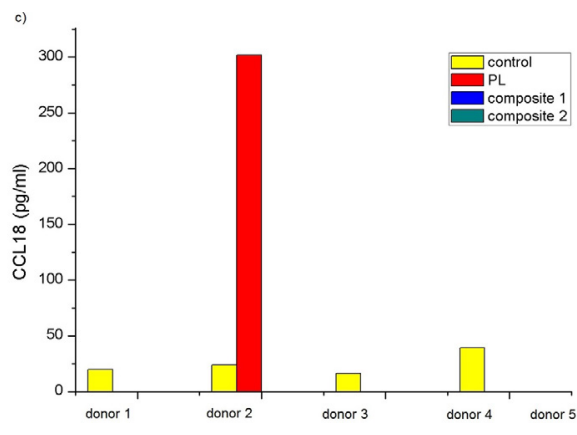

Figure 11: Effect of PL, composites 1, 2 on CCL18 production by primary human monocytes-derived macrophages: stimulation with IFNY.

TNF $\alpha$ is one of the earliest proinflammatory cytokines synthesized by monocytes and macrophages. TNF $\alpha$ acts upon macrophages as an autocrine and paracrine activator, i.e. in its presence the ability of macrophages for the eradication of microorganisms increases significantly. TNFa is a chemoattractant for macrophages; it stimulates the production of IL-1, prostaglandin E2, and granulocyte-macrophage colony-stimulating factor (GM-CSF). The local release of TNFa causes the active cell migration, phagocytosis stimulation, massive production of pro-inflammatory cytokines. The system release of TNF- $\alpha$ causes fever, hypotension and shock. This occurs during excessive activation of toll-like receptors on macrophages. This mediator was selected as the marker of the M1 activation marker for integrated risk assessment of the acute inflammatory immune reaction to studied polymeric materials. However, in the control and in the presence of PL, HA, composites 1, 2 secretion of TNFa is not fixed. Additionally, ELISA was conducted after addition of LPS and stimulation for 24 hours, but in this case the secretion of TNF $\alpha$ was observed only in the control, as well as in the control with IFN $\gamma$ (M1-activation); in the presence of materials $\mathrm{TNF} \alpha$, the expression is absent.

It can be assumed that the absence of TNFa secretion is due to the anti-inflammatory effect of IL-6, on the one hand, as described above. On the other hand, the cytokine network operates in such a way that in this case any proinflammatory mediator can duplicate the biological role of TNFa.

\section{Discussion}

As a result of the study, four types of samples: pure PL, HA, and composites on their basis with a content of components equal to 50:50, 75:25, were obtained. It was found that when mixing composites, chemical bonds and crystallographic characteristics of the individual components, which are connected to the sample through the Van der Waals and donor-acceptor bonds, are retained. It was shown that the proportion of PL and HA determined the dispersion and the crystallinity degree of materials. The material with ratio PL-HA=75/25 was characterized by the lowest degree of crystallinity equal to $20.5 \%$ and the average crystallite size of up to $28.8 \mathrm{~nm}$. Surface characteristics were determined by the quantitative ratio of components. The material containing $75 \mathrm{wt} . \%$ of polylactide was characterized by the increased surface roughness, and, in the series of samples, has the maximum dispersion component of surface energy that impacts biological characteristics.

The main factor in determining the dissolution rate in the physiological solution is the degree of samples' crystallinity, as well as the access of solvent to the polymer matrix loose areas. Composites 1 , 2 were able to form the calcium phosphate layer on their surface, and therefore they are capable to generate osseointegration. There is an active and uniform growth of the new layer on the surface of composite 2.

The authors also assessed viability of the immune system cells in the presence of PL, HA and composite materials. It was determined that the macrophages, in comparison with control, were the most viable in the presence of pure polylactide and composite 2. Upon cell interaction with PL, the authors have detected an expression of IL-6, which was not observed in the presence of the composites. In comparison with control, the concentration of CCL18 in the presence of PL did not change significantly. There was no expression of TNFa either in the control or in the presence of studied materials. This along with a minimum IL-6 secretion indicates a lack of inflammatory immune responses. Thus, materials containing 75 wt.\% of PL and $25 \%$ of HA are promising for its use as implant materials, because they are capable to substitute and restore bone tissue.

\section{Conclusion}

In the present study, polymer composite materials based on polylactide and hydroxyapatite were obtained. The formation of a new calcium-phosphate layer on the sample surface in a model SBF solution was studied. It was established that a uniform layer forms on the surface of a composite containing 75 weight $\%$ of polylactide. The viability of macrophages after cultivation in the presence of the obtained materials was also assessed. High cell viability is observed in the presence of pure polylactide and composite $\mathrm{PL} / \mathrm{HA}=75 / 25$. The results of ELISA showed that when cells with composite materials are cultured, the macrophage secretion of the pro-inflammatory cytokine IL-6 is minimal, and the secretion of TNFa is not recorded. However, significant secretion of the mediator - a marker of alternative macrophage activation, CCL 18, was not established either. These facts indicate a potential lack of pro-inflammatory properties in composites. In general, the studies that will be conducted in the future will help to uncover the mechanisms of the immune response of macrophages to composite materials for implantation and to develop approaches to the synthesis of innovative composites with tolerogenic properties.

\section{Conflict of Interest}

No

\section{Acknowledgment}

This research is supported by Tomsk State University Competitiveness Improvement Program «Molecular mechanisms of chronic inflammation in the progression of malignant neoplasms, cardiovascular diseases and implantation», No 8.1.16.2017 L.

\section{References}

1. Ratner BD (2004) Biomaterials science: an introduction to materials in medicine. Elsevier Academic Press, Boston, USA.

2. Chandra P, Yoo J, Lee $S$ (2015) Biomaterials in regenerative medicine: Challenges in technology transfer from science to process development. In: Atala A, Allikson J (eds) Translation Regenerative Medicine. Academic Press. pp. 151-167

3. Hench L, Jones J (2005) Biomaterials, artificial organs and tissue engineering Woodhead Publishing, UK.

4. Tang D, Tare R, Yang L, Williams D, Ou K, et al. (2016) Biofabrication of bone tissue: Approaches, challenges and translation for bone regeneration. Biomater 83: 363-382.

5. Niinomi M (2002) Recent metallic materials for biomedical applications. Metall Mater Trans A 33: 477-486

6. Breme H, Helsen J (1998) Metals as Biomaterials. Wiley, New York, USA.

7. Ratner B, Bryant S (2004) Biomaterials: Where we have been and where we are going. Annu Rev Biomed Eng 6: 41-75. 
Citation: Kurzina I, Churina Y, Shapovalova Y, Syusyukina V, Kzhyshkowska J (2018) Immunomodulatory Properties of Composite Materials Based on Polylactide and Hydroxyapatite. Bioceram Dev Appl 8: 109. doi: 10.4172/2090-5025.1000109

8. Levenberg S, Langer R (2004) Advances in tissue engineering: Current topics in developmental biology. Academic Press, New York, USA

9. Hench L (1998) Bioceramics. J Am Ceram Soc 81:1705-1728.

10. Kim H, Knowles J, Kim H (2004) Hydroxyapatite/poly(ع-caprolactone) composite coatings on hydroxyapatite porous bone scaffold for drug delivery. Biomater 25: 1279-1287.

11. Wang M (2003) Developing bioactive composite materials for tissue replacement. Biomaterials 24: 2133-2151.

12. Dang T, Nikkhah M, Memic A, Khademhosseini A (2015) Polymeric biomaterials for implanable prostheses, challenges in technology transfer from science to process development. In: Atala A, Allikson J (eds), Translation Regenerative Medicine. Academic Press. pp. 309-331

13. Tian H, Tang Zh, Zhuang X, Chen X, Jing X (2012) Biodegradable synthetic polymers: Preparation, functionalization and biomedical application. Prog in Polym Sci 37: 237-280

14. Jagur-Grodzinski J (2006) Polymers for tissue engineering, medical devices, and regenerative medicine Concise General Review of Recent Studies. Polym Advan Technol 17: 395-418.

15. Dorozhkin S (2011) Biocomposites and hybrid biomaterials based on calcium orthophosphates. Biomatter 1: 3-56

16. Hollister S, Maddox R, Taboas J (2002) Optimal design and fabrication of scaffolds to mimic tissue properties and satisfy biological constraints. Biomaterials 23: 4095-4103.

17. Scholz M, Blanchfield J, Bloom L (2011) The use of composite materials in modern orthopaedic medicine and prosthetic devices: a review.Compos SciTechnol 71: 1791-1803.

18. Zhou H, Touny A, Bhaduri S (2011) Fabrication of novel PLA/CDHA bionanocomposite fibers for tissue engineering applications via electrospinning. J Master Sci Mater Med 22: 1183-1193.

19. Gupta B, Revagade N, Hilborn J (2007) Poly (lactic acid) fiber: an overview. Prog Polym Sci 32: 455-482.

20. Goswami J, Bhatnagar N, Mohanty S, Ghosh A (2013) Processing and characterization of poly(lactic acid) based bioactive composites for biomedical scaffold application. eXPRESS Polym Lett 7: 767-777.

21. Uhrich K, Cannizzaro S, Langer R, Shakesheff K (1999) Polymeric system for controlled drug release. Chem Rev 99: 3181-3198

22. Mucalo M (ed), (2015) Hydroxyapatite (Hap) for Biomedical Applications Woodhead Publishing, UK.
23. Ben-Nissan B (2014) Advances in calcium phosphate biomaterials. Spinger.

24. Fujii S, Miyanari Y, Nishimura T, Yokoyama Y, Hamasaki S, et al. (2013) In vitro degradation of hydroxyapatite nanoparticle-coated biodegradable microspheres. Polym Degrad Stabil 98: 377-386.

25. Peng F, Yu X, Wei M (2011) In vitro cell performance on hydroxyapatite particles/poly (L-lactic acid) nanofibrous scaffolds with an excellent particle along nanofiber orientation. Acta Biomater 7: 2585-2592.

26. Ignjatovic N, Uskokovic D (2004) Synthesis and application of hydroxyapatite/ polylactide composite biomaterial. Appl Surf Sci 238: 314-319.

27. Bartolo P, Kruth J, Silva J (2012) Biomedical production of implants by additive electro-chemical and physical processes. CIRP Annals - Manufacturing Technology 61: 635-655.

28. Jones C, Arns C, Hutmacher D, Milthorpe BK, Sheppard AP, et al. (2009) The correlation of pore morphology, interconnectivity and physical properties of 3D ceramic scaffolds with bone in growth. Biomaterials 30: 1440-1451.

29. Kasuga T, Ota Y, Nogami M, Abe Y (2001) Preparation and mechanica properties of polylactic acid composites containing hydroxyapatite fibers. Biomaterials 22: 19-23.

30. Diao H, Si Y, Zhu A, Ji L, Shi H (2012) Surface modified nano-hydroxyapatite/ poly(lactide acid) composite and its osteocyte compatibility. Mater Sci Eng C 32: 1796-1801.

31. Honga Zh, Qiua X, Suna J, Denga M, Chena X, et al. (2004) Grafting polymerization of L-lactide on the surface of hydroxyapatite nano-crystals. Polymer 45: 6699-6706.

32. Hong Zh, Zhang P, He C, Qiu X, Liu A, et al. (2005) Nano-composite of poly (L-lactide) and surface grafted hydroxyapatite: Mechanical properties and biocompatibility. Biomaterials 26: 6296-6304.

33. Nampoothiri K, Nair N, John R (2010) An overview of the recent developments in polylactide (PLA) research. Bioresource Technol 101: 8493-8501.

34. Rasskazova L, Korotchenko N, Zeer G (2013) Microwave synthesis of hydroxyapatite and physicochemical study of its properties. Russ J Appl Chem 86: 691-695.

35. Kokubo T (1991) Bioactive glass ceramics: properties and applications Biomaterials 12: 155-163.

36. Popova A, Kzhyshkowska J, Nurgazieva D, Goerdt S, Gratchev A (2011) Proand anti-inflammatory control of M-CSF mediated macrophage differentiation. Immunobiology 216: 164-172. 